\title{
Acknowledgment of Ad Hoc Reviewers
}

The Editor and Associate Editors gratefully acknowledge the following reviewers, who reviewed manuscripts for the 2016 volume.

\begin{tabular}{|c|c|}
\hline Alberto Acerbi & Robert Bohm \\
\hline Hajo Adam & Vanessa Bohns \\
\hline Roee Admon & Steve Boker \\
\hline Christopher Agnew & Walter R. Boot \\
\hline Woo-Young Ahn & Heather Bortfeld \\
\hline Lara Beth Aknin & Roberto Bottini \\
\hline William Alexander & Ty Boyer \\
\hline Mark Alicke & Charles J. Brainerd \\
\hline Brian A. Anderson & Neil Robert Bramley \\
\hline Michael C. Anderson & Mark Brandt \\
\hline Tim Andrews & Marcel Brass \\
\hline Evan Apfelbaum & Todd Braver \\
\hline Matthew Apps & Mara Breen \\
\hline Robert Ariel & C. Miguel Brendl \\
\hline Aiden Arnold* & Christine Brennan \\
\hline Adam Aron & Neil Brewer \\
\hline Joseph L. Austerweil & Melanie Brewster \\
\hline Hillel Aviezer & Pablo Brinol \\
\hline Judith Avrahami & James Broadway \\
\hline \multirow[t]{2}{*}{ Peter Ayton } & David Broockman \\
\hline & Gordon Brown \\
\hline Matthijs Baas & Sarah Brown-Schmidt \\
\hline Harald Baayen & Emile Gabriel Bruneau \\
\hline Gi-Yeul Bae & Tad Brunye \\
\hline Bahador Bahrami & Christopher Bryan \\
\hline Emily Balcetis & Greg Bryant \\
\hline Bernard Balleine & Emma Buchtel \\
\hline Moshe Bar & Berno Bucker* \\
\hline Yoav Bar-Anan & David Budescu \\
\hline John Bargh & Marc Jens Buehner \\
\hline Jonathan Baron & Melissa Buelow \\
\hline Shira Baror* & Simona Buetti \\
\hline Brock Bastian & Julie Bugg \\
\hline Christine Bastin & Tyler J. Burleigh* \\
\hline Roy Baumeister & Jerome Busemeyer \\
\hline Karl-Heinz T. Bauml & Laura Bustamante* \\
\hline Karin Bausenhart & Fabrizio Butera \\
\hline Andrew P. Bayliss & Andrew C. Butler \\
\hline Paul Bays & David Buttelmann \\
\hline Stefanie I. Becker & Ruth Byrne \\
\hline \multicolumn{2}{|l|}{ Denise R. Beike } \\
\hline Artem Belopolsky & Jimmy Calanchini \\
\hline Michael Beran & Christine Caldwell \\
\hline Yoella Bereby-Meyer & Joan Camprodon \\
\hline Howard Berenbaum & Peter Carnevale \\
\hline Sheri Berenbaum & Peter Carruthers \\
\hline Kirsten Bergmann & Evan C. Carter* \\
\hline Elliot T. Berkman & Evelyn Carter* \\
\hline Bennett I. Bertenthal & McKell Carter \\
\hline Marco Bertramini & Eugene M. Caruso \\
\hline Sudeep Bhatia & Paulo Carvalho \\
\hline Erik Bijleveld & Beth Casey \\
\hline Merim Bilalic & Alan Castel \\
\hline Patrick Bissett & Monica Castelhano \\
\hline R. Thora Bjonsdottir* & Anne Castles \\
\hline Mark Blair & Kyle Cave \\
\hline Neeltje Blankenstein & Joseph Cesario \\
\hline Ned Block & Ya-Ning Chang \\
\hline Susan Bluck & Catherine Chase \\
\hline
\end{tabular}

Michael W. L. Chee

Jacqueline M. Chen

Ken Cheng

Paolo Cherubini

Nicholas Chevalier

Heather Cipoletti

Claudia Civai* $^{*}$

Jasmin Cloutier

Andrew Cohen

Adam Cohen

Geoff Cole

Anne Collins

Luanna Colloca

Roberto Colom

Paul Condon

Andrew Connolly

Andrew Conway

Richard P. Cooper

Jonathan Corbin

Joshua Correll

Fintan John Costello

Nelson Cowan

Cathy Cox

Fergus I. M. Craik

Sarah C. Creel

Davide Crepaldi

Amy Criss

Hugo Critchley

Eveline Crone

Geoff Cumming

Denise Cummins

Kim Curby

Ryan Curl*

Fiery Cushman

J. Cooper Cutting

Junyi Dai

Hengchen Dai

Kirsten Dalrymple

Michael Dambacher

Nicolas Davidenko

Catherine Davies

Elizabeth Davis

Colin Davis

Amy Dawel

Carsten de Dreu

Jan W de Fockert

Maria de Hevia

Peter De Jong

Benedetto De Martino

Sanne de Wit

Joseph DeGutis

Jasmine DeJesus

Michael L. DeKay

Fabio Del Missier

Andrew Delamater

Heath Demaree

Rutvik Desai

Peter Descioli
Jason Deska*

Nathan DeWall

Adele Diederich

David Dignath

Laura Dilley

Annie Ditta*

James Dixon

Peter Dixon

Chad Dodson

Brent Donnellan

Ron Dotsch

Ana Draghici*

Nicolas Dumay

John Dunlosky

Elizabeth Dunn

David A. Dunning

Nicholas Duran

Wouter Duyck

Alice Eagly

Jane Ebert

Andreas Eder

Tobias Egner

Arne Ekstrom

Hillary Elfenbein

Andrew Elliot

Emily M. Elliott

Kristen Elmore

Nicholas Epley

Ido Erev

Kimmo Eriksson

Gennady Erlikhman

Carsten Erner

Zachary Estes

Anthony M. Evans

Simon Evans

Ellen Evers

Youssef Ezzyat

Aidan Feeney

Helen Feigin*

Heather Ferguson

Philip Michael Fernbach

Daniel Fessler

Matt Field

Bernd Figner

József Fiser

Shelley B. Flagel

Jonathan Isaac Flombaum

Charles Folk

Cyrus K. Foroughi

Kenneth I. Forster

Jens Förster

Tom Foulsham

Craig Fox

Mary Frame

Gregory Francis

Ana Franco-Watkins

Scott Fraundorf 
Jonathan Freeman

Jon Freeman

David Freestone

Alexandra Freund

Ori Friedman

Ron Friedman

Naomi Friedman

Justin Friesen

Philip A Gable

Sam Gaertner

Jeff Galak

Yoav Ganzach

Danilo Garcia

Stephen M. Garcia

Benjamin Gardner

Isabel Gauthier

Janet Geipel

Guido H. E. Gendolla

Silvia Gennari

Walter Gerbino

Samuel J. Gershman

Will Gervais

Gerd Gigerenzer

Daniel Gilbert

Thomas Gilovich

Andreas Gloeckner

Judith Gluck

Stephen D. Goldinger

Robert Goldstone

Agnieszka Golec de Zavala

Cleotilde Gonzalez

Claudia Gonzalez-Vallejo

Geoffrey Goodwin

Yonatan Goshen-Gottstein

Roland Grabner

Susan Graham

Kurt Gray

Shawn C. Green

Donald Green

Katherine Greenaway

Tobias Greitemeyer

Nurit Gronau*

Scott D. Gronlund

Blair Gross

Igor Grossmann

Marc Guitart-Masip

Michaela Gummerum

Daniel E. Gustavson

Martin Hagger

Nao Hagiwara

Ulrike Hahn

Jamin Halberstadt

Zach Hambrick

Antonia Hamilton

James A. Hampton

Jochim Hansen

Paul L. Harris

Tyler L. Harrison

Cate Hartley

Mark Haselgrove

Lynn Hasher

Nick Haslam
John Hattie

Olaf Hauk

David J. Hauser

Brett K. Hayes

Eliot Hazeltine

Michael Karl Healey*

Andrew Heathcote

Steven Heine

Agneta Herlitz

Stefan Herzog

Ursula Hess

Cecilia Heyes

Clayton Hickey

Joshua Hicks

Phil Higham

Aline Hitti

Guy Hochman

Sarah Hodges

Michael Hoerger

Erika Hoff

Ulrich Hoffrage

Robin M. Hogarth

John Holmes

Clay Holroyd

Bernhard Hommel

William S. Horton

Marc Howard

Markus Huff

Kurt Hugenberg

Sean Hughes

Cendri Hutcherson

Mandy Hütter

Hyisung C. Hwang*

Roland Imhoff

Yoel Inbar

Helene Intraub

Michael Inzlicht

Heather Iriye*

Jess Irons

Keise Izuma

T. Florian Jaeger

Sara Jahfari*

Randy Jamieson

Petr Janata

Yoonhee Jang

Debra Jared

Andrew Jarosz

Andreas Jarvstad

Lisa Jeffries

Veronika Job

Clinton L. Johns

Michael Jones

Fredrik Jonsson

Jillian Jordan

Alexander Jordan

Peter Juslin

Joe Kable

Zsuzsa Kaldy

Arvid Kappas

Yaakov Kareev

Uma R. Karmarkar
Todd Kashdan

Yoshihisa Kashima

Jun Kawahara

Frank Keil

David Kellen

Dacher Keltner

Alice Kim

Nancy S. Kim

Laura King

Alan Kingstone*

Shinobu Kitayama

Roberta Klatzky

K. Christoph Klauer

Reinhold Kliegl

Joshua Knobe

Guenther K. Knoblich

Melissa Koenig

Wouter Kool

Sander Koole

Jonathan Koppel

Asher Koriat

Maryam Kouchaki

Ian Krajbich

Max Krasnow

Ruth Krebs

Jodi Kreiman

Anne Krendl

Amy Krosch

Dario Krpan

Joachim I. Krueger

Joachim I. Krueger

Daniel Kruger

Eva Krumhuber

Jennifer Kubota

Victor Kuperman

Kenneth Kurtz

Peter Kvam

Jessica Kwong

Thomas Lachmann

Daniel Lakens

Sarah Ariel Lamer

David Lancy

Justin Frederick Landy

Michael S. Landy

Maria Larsson

Mike Le Pelley

Pontus Leander

Sang Ah Lee

Joanna Lee

Jo-Anne LeFevre

Cristine Legare

Bernhard Leidner

Tomás Lejarraga

Gareth Leng

Linda J. Levine

Stephen Lewandowsky*

Nira Liberman

Heinrich Rene Liesefeld*

Nichole Lighthall

Mimi Liljeholm

Richard Liu

Evan Livesey

Jeffrey Lockman
Chris Loersch

Gordon D. Logan

Tania Lombrozo

Andrew Lotto

Matthew L. Lowder*

Shenghua Luan

Richard E. Lucas

Gigi Luk

Wenbo Luo

Gary Lupyan

Caroline Lustenberger

John G. Lynch

Christine Ma-Kellams

Anne Maass

Maryellen MacDonald

Arien Mack

Michael Mack

Colin M. MacLeod

C. Neil Macrae

Joseph Magliano

Sam Maglio

Bradford Mahon

Tal Makovski

George L. Malcolm

Bertram F. Malle

Eric Mandelbaum

Antony Manstead

Petra Marcotti*

Elizabeth Margulis

Doug Markant

Justin Martin

Andrea Mason

Michael E. J. Masson

Rui Mata

Kyle Mathewson

Sebastiaan Mathot

David Matsumoto

William Matthews

Mark Mattson

Iris Mauss

Richard E. Mayer

Ulrich Mayr

Samuel T. McAbee

Dawn McBride

James McClelland

Samuel M. McClure

Michael McCullough

Joseph McGuire

James McQueen

Jennifer McVay

Daniel Meegan

Matthias Mehl

Pranjal Mehta

Nachshon Meiran

Monica Melby-Lervag

Edgar C. Merkle

Tom Meyvis

Catherine D. Middlebrooks*

Petar Milin

Greg Miller

Ralph R. Miller

Mark Mills

Marina Milyavskaya 
Dan Mirman

Vlad Miskovic

Daniel Mochon

Daniel C. Molden

Ludwin Molina

Cathy Mondloch

Andrew E. Monroe

Margo Monteith

Chris Moore

Adam Moore

Cathleen Moore

Don Moore

James Moore

Katherine S. Moore

Timothy Moran

Sylvia Morelli

Carey K. Morewedge

Tuuli Morrill

Hermann Josef Muller

Tim Mullett

Kou Murayama

Gregory L. Murphy

Micah M. Murray

Wayne Murray

Sebastian Musslick ${ }^{*}$

Thomas Mussweiler

Kate Nation

Derek Evan Nee

Leif Nelson

Ben Newell

Charles Nichols

Mark Nieuwenstein

Mante Nieuwland

Loran Nordgren

Michael Norton

Ludmila Nunes

Ed O'Brien

Klaus Oberauer

Nicole Oei

Philipp Opitz*

Allen Osman*

Frederick Oswald

Ross Otto

David Over

Daphna Oyserman

Thorsten Pachur

Steven Pan*

Anna Papafragou

Megan H. Papesh

Esther Papies

Soojin Park

Philip Pärnamets

Erika Patall

Kristin Pauker

Philip Pavlik

Jessica D. Payne

Diane Pecher

Trevor Penney

Amy Perfors

Tyler Perrachione

Anat Perry
Steve Petrill

John V. Petrocelli

Kathy Pezdek

Frederick L. Philippe

L. Taylor Phillips

Jared Piazza

David Pillemer

Margarida Pitaes

Jason Plaks

Timothy J. Pleskac

Endel Põder

Fenna Poletiek

Evan Polman

James Pomerantz

Bob Port

Emmanuel Pothos

Catherine Preston

Tila Pronk

Travis Proulx

Candace Raio

Gerardo Ramirez

Richard Ramsey

David Rand

Daniel Randles

Gary Raney

Kathy Rastle

Birgit Rauchbauer

Jane Raymond

Stephen John Read

Rolf Reber

Thomas Redick

Stephen Reicher

Jamie Reilly

Valerie Reyna

Marjorie Rhodes

Matthew Rhodes

Daniel C. Richardson

Timothy Rickard

Timothy J. Ricker

Jörg Rieskamp

Evan Risko

Ilana Ritov

Grace Rivera

Rachel Robbins

Debi Roberson

Ian Robertson

Timothy Rogers

Doug Rohrer

Zachary Roper

David Ross

Mandy Rossignol

Klaus Rothermund

Yuval S. Rottenstreich

Benjamin Margolin Rottman

Paul Rozin

Jay Rueckl

Shirley-Ann Rueschemeyer

Nick Rule

Nikol Rummel

James A. Russell

Jay Edward Russo

Abe Rutchick

Eric Ruthruff
Pertti Saariluoma

Timothy Salthouse

Jason Samaha

Gregory Samanez-Larkin

Adam Sanborn

Alan Sanfey

Joseph Sanfilippo*

Kai Sassenberg

Marian Sauter"

E. Glenn Schellenberg

Dan Schley

Simone Schnall

Iris Schneider

Abigail A. Scholer

Marc Scholten

Tom Schonberg

Jonathan Schooler

Lael Schooler

Juliana Schroeder

Laura Schulz

Christin Schulze

Stephan Schwan

Barry Schwartz

Oliver Schweickart

Nick Schweitzer

Sophie Scott

Michael Scullin

Per B. Sederberg

Constantine Sedikides

Paul Seli

Andrea Sell

Gun Semin

Thomas Serre

Alexander Shackman

Nicholas Shakeshaft

Amitai Shenhav

Zach Shipstead

Kristin Shutts

Heida Maria Sigurdardottir

Rita Silva*

Mark Simmonds

Joseph Simmons

Uri Simonsohn

Itamar Simonson

John J. Skowronski

Willem Sleegers*

Vladimir Sloutsky

Daniel Smilek

Steven M. Smith

Michael Smithson

Peter Sokol-Hessner

Mitch Sommers

Maarten Speekenbrink

Michael J. Spivey

Susan Sprecher

Sanjay Srivastava

Peggy St Jacques

Christoph Stahl

Aimee Stahl

Tom Stanley

Robert Sternberg

Neil Stewart

Benjamin Storm

Julia Strand
Jie Sui

Caterina Suitner

Kwanho Suk

Claire Sulmont-Rosse

Abigail Sussman

Shinsuke Suzuki

Lee Swanson

Tim Sweeny

Benjamin Swets

Diane Swick

Karl Szpunar

Marcus Taft

Thomas Talhelm

James W. Tanaka

Karl Halvor Teigen

Josh Tenenbaum

Katy Thakkar

Anna Theakston

Rachel Theodore

Rick Thomas

Robin D. Thomas

Mark Tiede

Jason Tipples

Andrew R. Todd

Peter M. Todd

Claudia Toma

Andrew Tomarken

Negin Toosi

Sascha Topolinski

Keith Topping

Joseph Toscano

James Townsend

Sophie Trawalter

Matthew Traxler

Michael Treadway

Jeremy Tree

Rebecca Treiman

Dries Trippas*

Jennifer Trueblood

Jeanne Tsai

Philip Tseng

Konstantinos Tsetsos

Jonathan Garrett Tullis

Joshua Tybur

Eric Uhlmann

Gonzalo Urcelay

Oleg Urminsky

Piercarlo Valdesolo

Gaëlle Vallée-Tourangeau

Sofia Vallila-Rohter

Patty Van Cappellen

Eric van Dijk

Michiel van Elk

Don van Ravenzwaaij

Hedderik van Rijn

Mark Van Vugt

Andre Vandierendonck

Eric Vanman

Trish Varao-Sousa

Evie Vergauwe

Sara Verosky 
Gabriella Vigliocco

David Vinson

Melissa Vo

Katharina von Kriegstein

Joachim Vosgerau

Ed Vul

Monica Wadhwa

Michaela Waenke

Johan Wagemans

Eric-Jan Wagenmakers

Tor Wager

Lukasz Walasek

Jeffrey Walczyk

Drew Walker

Eva Walther
Geoff Ward

Sarah Ward*

Florian Waszak

Katsumi Watanabe

Adam Waytz

Annie Weldon*

Gary Wells

Dirk Wentura

Michael Wenzel

J. Andrew Westbrook*

Jacob Westfall

Corey White

Alex L. White

Peter A. White

Andrew Whiten

David Whitney
Michael Wiedmann*

Martin Wiener

Reinout Wiers

Jennifer Wiley

Anne Wilson

Christine Wilson-Mendenhall

Piotr Winkielman

Jessica K. Witt

Bernd Wittenbrink

Bianca Wittmann

Conny Wollbrant

Karl-Andrew Woltin

Wendy Wood

Dan Wright

Anthony A. Wright

Dirk Wulff
Erte Xiao

Takashi Yamauchi

David Yeager

Eldad Yechiam

Eiling Yee

Shuli Yu

Daniel Yudkin*

Paree Zarolia

Peter Zeidman

Jason Zevin

Jennifer Zuk

* Denotes a reviewer who co-reviewed under the supervision of a primary reviewer.
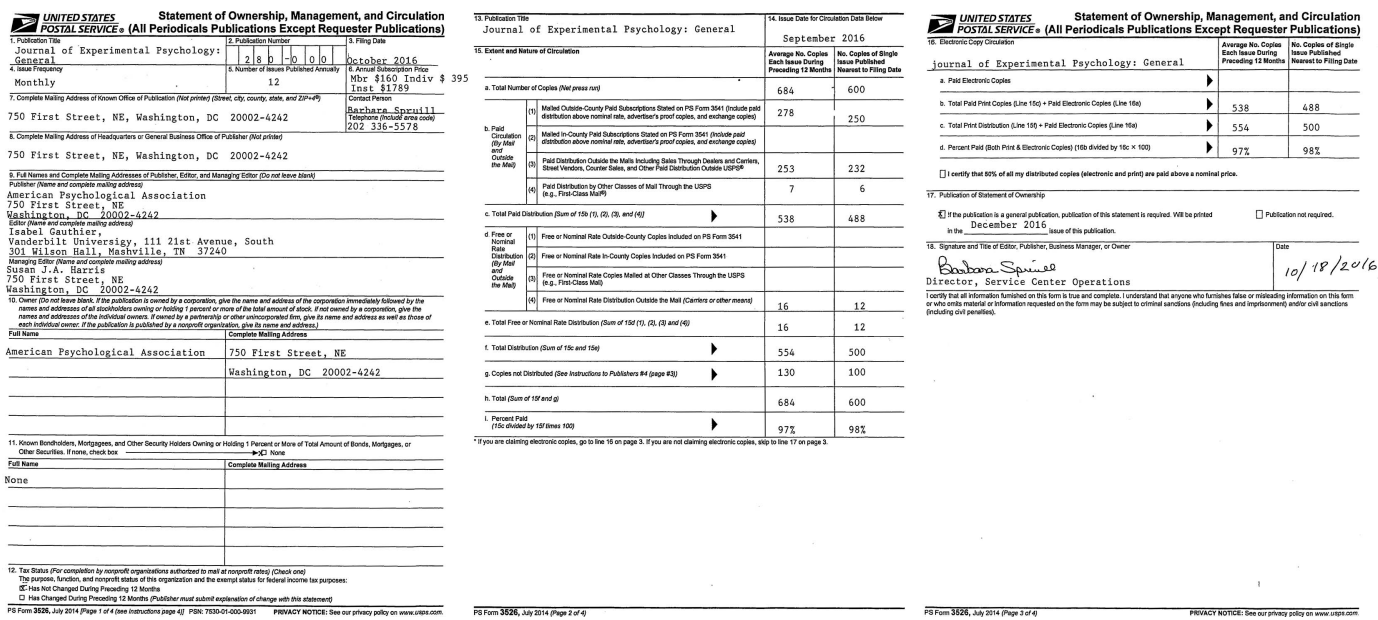